\title{
On M-9-branes and their dimensional reductions
}

\author{
Takeshi Sato ${ }^{a}$ \\ ${ }^{a}$ Institute for Cosmic Ray Research, University of Tokyo, \\ 5-1-5 Kashiwanoha, Kashiwa-shi, Chiba-ken, 277-8582, Japan
}

The M-9-brane Wess-Zumino action is constructed, and by using it, consistency of the relation of p-branes for $p \geq 8$, suggested on the basis of superalgebra, is discussed.

\section{Introduction}

M-theory is a candidate for a unified theory of particle interactions and is conjectured to be the 11-dimensional (11D) theory which gives 5 perturbative 10-dimensional (10D) string theories in different kinds of limits. In discussing properties of these theories, $(p+1)$-dimensional objects, called p-branes, play many crucial roles, so, it is important to clarify what kinds of branes exist in each of the theories. Brane scan via superalgebra is one of the methods to discuss them, by which BPS branes possible to exist in the theories are predicted[1] [2] (see also [3]). For the case $p \leq 7$, all the p-branes predicted to exist in M-, IIA and IIB string theories, have corresponding solutions in each of their supergravity theories. As for the p-branes with $p \geq 8$, however, there is a problem, as we will explain later. In this work we will discuss these branes, since p-branes with $p \geq 8$ are very important in that $\mathrm{M}$ - and string theories with 16 supercharges are expected to be constructed by using these branes (see ref. 4 and references therein).

To be concrete, one kind of 9-brane is suggested to exist in M-theory [1], one kinds of 8-brane and 9-brane are predicted in IIA, and two kinds of 9-branes are in IIB]1]. The first one is called "M9-brane", and the others are called or identified with D-8-brane, NS-9A-brane, D-9-brane and NS9B-brane, respectively, based on the consideration of their kind of charges. Taking into account the dimensions and the duality relations of the theories, the relation of the p-branes for $p \geq 8$, suggested based on superalgebra, is represented as Figure 1[1].

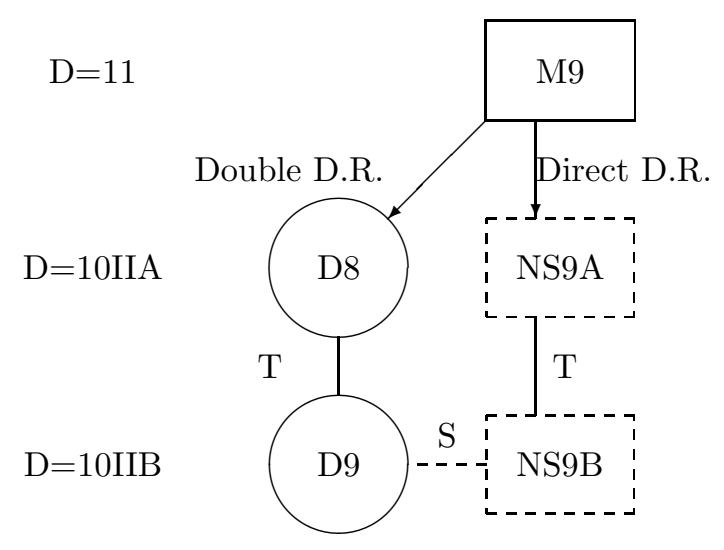

Figure 1. The relation of p-branes with $p \geq 8$. (D.R. denotes dimensional reduction.)

However, there is the following problem with the M-9-brane, or 11D origins of the D-8-brane solution and massive IIA supergravity (SUGRA). The BPS D-8-brane arising in the IIA string, actually, has a corresponding solution not in the usual IIA but in the massive IIA SUGRA with

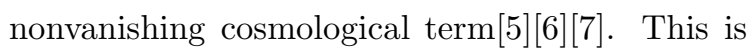
because a BPS D-8-brane in 10 dimensions is a domain wall with some electric charge of a RR 9-form gauge field, giving rise to a constant field strength, whose dual we denote as a mass pa- 
rameter $m$. This field strength contributes to the action as a cosmological constant $-m^{2} / 2$. In other words, such domain wall solutions cannot be constructed without cosmological term. In 11 dimensions (11D), however, no deformation to include a cosmological term is allowed if Riemannian geometry and covariant action are assumed [8]. Thus, there is no naive M-9-brane solution in 11D, and the origin of the D-8-brane and massive IIA SUGRA are still unclear.

There are several approaches to solve this problem and one of them is "massive 11D theory" [9]. This is a trial theory, constructed on the basis of the idea that the problem may imply the need to modify the framework of 11D SUGRA. Suppose a Killing isometry is assumed in the 11D background. Then, the no-go theorem is avoided and the massive 11D theory, which is written in terms of an 11-dimensional theory at least formally, can be defined; it gives the 10D massive IIA SUGRA on dimensional reduction along the isometry direction (which is parametrized by the coordinate $z$ ), and gives usual 11D SUGRA in the massless limit $m \rightarrow 0$ if the dependence of the fields on $z$ is restored. Moreover, the M-9-brane solution, i.e. the solution which gives a D-8-brane solution on the dimensional reduction along $z$, is obtained in this theory [10]. We note that only the bosonic sectors have been discussed in this massive 11D theory, though its bulk theory is called "super" gravity. We also note that the isometry direction is interpreted as a compactified direction like $S^{1}$, and the M-9-brane is considered to be wrapped around it 10 . We follow the above idea and study the relation of branes within this framework.

To be concrete, we will discuss the relation from the viewpoint of worldvolume effective action (WVEA). In fact, almost all of the WVEA's of the branes have been already obtained. However, only the Wess-Zumino (WZ) term of the M9-brane $S_{M 9}^{W Z}$ has not yet been obtained. So, as for the WZ terms, consistency of the relation in Figure 1 has not been established. In other words, even within this framework, the consistency of the relation of the branes has not been established yet. The purpose of this work is to construct $S_{M 9}^{W Z}$ and to examine the consistency of the relation of the p-branes with $p \geq 8$ from the viewpoint of worldvolume effective action.

The concrete procedures are as follows: First, we introduce a 10 -form gauge potential into the theory consistently because the M-9-brane is expected to couple to it. Then, we construct the M-9-brane WZ action using the 10-form and examine the consistency of the action in certain two ways. Finally, we investigate two kinds of dimensional reductions of the action.

This talk is based on work [11,12].

\section{The M-9-brane WZ action and its di- mensional reductions}

First, we briefly review the massive 11D SUGRA[9]. The bosonic field content is the same as that of the usual (massless) 11D SUGRA: the metric $\hat{g}_{\mu \nu}$ and a 3 -form gauge potential $\hat{C}_{\mu \nu \rho}$. In this theory these fields are required to have a Killing isometry, i.e., $\mathcal{L}_{\hat{k}} \hat{g}_{\mu \nu}=\mathcal{L}_{\hat{k}} \hat{C}_{\mu \nu \rho}=0$ where $\mathcal{L}_{\hat{k}}$ indicates a Lie derivative with respect to a Killing vector field $\hat{k}^{\mu}$. (We fix the coordinates so that $\hat{k}^{\mu}=\delta^{\mu z}$.) The infinitesimal gauge transformations of the fields are defined as

$$
\begin{gathered}
\delta \hat{g}_{\mu \nu}=-m\left[\hat{\lambda}_{\mu}\left(i_{\hat{k}} \hat{g}\right)_{\nu}+\hat{\lambda}_{\nu}\left(i_{\hat{k}} \hat{g}\right)_{\mu}\right], \\
\delta \hat{C}_{\mu \nu \rho}=3 \partial_{[\mu} \hat{\chi}_{\nu \rho]}-3 m \hat{\lambda}_{[\mu}\left(i_{\hat{k}} \hat{C}\right)_{\nu \rho]}
\end{gathered}
$$

where $\left(i_{\hat{k}} T_{\mu_{1} \cdots \mu_{r-1}}^{(r)}\right) \equiv \hat{k}^{\mu} T_{\mu_{1} \cdots \mu_{r-1} \mu}^{(r)}$ for a field $T^{(r)} . \hat{\chi}$ is the infinitesimal 2-form gauge parameter, $\hat{\lambda}$ is defined as $\hat{\lambda}_{\mu} \equiv\left(i_{\hat{k}} \hat{\chi}\right)_{\mu}$, and $m$ is a constant mass parameter. Then, a connection for the massive gauge transformations should be introduced. The new total connection takes the form $\hat{\Omega}_{a}^{b c}=\hat{\omega}_{a}^{b c}+\hat{K}_{a}^{b c}$ where $\hat{\omega}_{a}^{b c}$ is a usual spin connection and $\hat{K}$ is given by

$\hat{K}_{a}^{b c}=\frac{m}{2}\left[\hat{k}_{a}\left(i_{\hat{k}} \hat{C}\right)^{b c}+\hat{k}_{b}\left(i_{\hat{k}} \hat{C}\right)^{a c}-\hat{k}_{c}\left(i_{\hat{k}} \hat{C}\right)^{a b}\right] .(2)$

The 4-form field strength $\hat{G}^{(4)}$ of $\hat{C}$ is defined as

$$
\begin{aligned}
\hat{G}_{\mu \nu \rho \sigma}^{(4)}=4 D_{[\mu} \hat{C}_{\nu \rho \sigma]} & \equiv 4 \partial_{[\mu} \hat{C}_{\nu \rho \sigma]} \\
& +3 m\left(i_{\hat{k}} \hat{C}\right)_{[\mu \nu}\left(i_{\hat{k}} \hat{C}\right)_{\rho \sigma]}
\end{aligned}
$$

where $D_{\mu}$ denotes the covariant derivative. Then, $\hat{G}^{(4)}$ transforms covariantly as $\delta \hat{G}_{\mu \nu \rho \sigma}^{(4)}=$

\footnotetext{
${ }^{1}$ We use $a, b, \cdots$ for local Lorentz indices.
} 
$4 m \hat{\lambda}_{[\mu}\left(i_{\hat{k}} \hat{G}^{(4)}\right)_{\nu \rho \sigma]}$, which implies that $\delta\left(\hat{G}^{(4)}\right)^{2}=$ 0 .

The action of the massive 11D SUGRA is

$$
\begin{gathered}
\hat{S}_{0}=\frac{1}{\kappa} \int d^{11} x\left[\sqrt { | \hat { g } | } \left\{\hat{R}-\frac{1}{2 \cdot 4 !}\left(\hat{G}^{(4)}\right)^{2}\right.\right. \\
\left.+\frac{1}{2} m^{2}|\hat{k}|^{4}\right\}+\frac{\hat{\epsilon}^{\mu_{1} \cdots \mu_{11}}}{(144)^{2}}\left\{2^{4} \partial \hat{C} \partial \hat{C} \hat{C}\right. \\
\left.\left.+18 m \partial \hat{C} \hat{C}\left(i_{\hat{k}} \hat{C}\right)^{2}+\frac{3^{3}}{5} m^{2} \hat{C}\left(i_{\hat{k}} \hat{C}\right)^{4}\right\}_{\mu_{1} . . \mu_{11}}\right]
\end{gathered}
$$

where $\kappa=16 \pi G_{N}^{(11)}$ and $|\hat{k}|=\sqrt{-\hat{k}^{\mu} \hat{k}^{\nu} \hat{g}_{\mu \nu}}$. This action is invariant (up to total derivative) under (11), and its dimensional reduction along $z$ gives the bosonic part of 10D massive IIA SUGRA action.

Now, let us introduce a 10 -form gauge potential $\hat{A}^{(10)}$. Following the case of the 9 -form potential in 10D IIA theory $[7]$, we promote the mass parameter $m$ to a scalar field $\hat{M}(x)$, and add the term

$\frac{1}{\kappa} \int d^{11} x \frac{11}{11 !} \hat{\epsilon}^{\mu_{1} \cdots \mu_{11}} \hat{M}(x) \partial_{\left[\mu_{1}\right.} \hat{A}_{\left.\mu_{2} \cdots \mu_{11}\right]}^{(10)}$.

to the action $\hat{S}_{0}$ to introduce $\hat{A}^{(10)}$ as a Lagrange multiplier for $\hat{M}(x)=m$. We note that $\hat{A}^{(10)}$ also satisfies $\mathcal{L}_{\hat{k}} \hat{A}^{(10)}=0$, which means that $\hat{A}^{(10)}$ with no $z$ index does not appear in this theory. Then, the action is invariant under (1) if the massive gauge transformation of $\hat{A}^{(10)}$ is defined as

$$
\begin{array}{r}
\delta\left(i_{\hat{k}} \hat{A}^{(10)}\right)_{\mu_{1} \cdots \mu_{9}}=-\sqrt{|\hat{g}|} \hat{\epsilon}_{\mu_{1} \cdots \mu_{9} z} \\
\cdot\left[-\hat{g}^{\mu \mu^{\prime}} \hat{g}^{\nu \nu^{\prime}}\left(2 \partial_{\left[\mu^{\prime}\right.} \hat{k}_{\left.\nu^{\prime}\right]}-\hat{M}|\hat{k}|^{2}\left(i_{\hat{k}} \hat{C}\right)_{\mu^{\prime} \nu^{\prime}}\right) \hat{\lambda}_{\nu}\right. \\
\left.+\frac{1}{2} \hat{G}^{(4) \mu \nu \rho \sigma}\left(i_{\hat{k}} \hat{C}\right)_{\nu \rho} \hat{\lambda}_{\sigma}\right] \\
-\frac{9 !}{48}\left[\partial \hat{C}\left(i_{\hat{k}} \hat{C}\right)^{2} \hat{\lambda}+\frac{\hat{M}}{4}\left(i_{\hat{k}} \hat{C}\right)^{4} \hat{\lambda}\right]_{\mu_{1} \cdots \mu_{9}} .
\end{array}
$$

Now, we discuss the construction of $S_{M 9}^{W Z}$ using $\hat{A}^{(10)}$. However, the gauge invariant WZ action cannot be constructed straightforwardly. The reason is as follows: Since a M-9-brane couples to $\hat{A}^{(10)}, S_{M 9}^{W Z}$ contains the term

$$
\begin{aligned}
& \left.S_{M 9}^{W Z}\right|_{9 \text { form }}=\frac{T_{M 9}}{9 !} \int d^{9} \xi \epsilon^{i_{1} . . i_{9}} \\
& \times \partial_{i_{1}} X^{\hat{\mu}_{1}} . . \partial_{i_{9}} X^{\mu_{9}}\left(i_{\hat{k}} \hat{A}^{(10)}\right)_{\mu_{1} . . \mu_{9}}
\end{aligned}
$$

where $\xi^{i}(i=0, . ., 8)$ are worldvolume coordinates of the brane and $\hat{X}^{\mu} \quad(\mu=0, . ., 9)$ are embedding coordinates. Suppose we consider the massive gauge transformation of (7). Then, we can see that the contribution of the first bracket of the r.h.s. of (6) to the variation of (7) cannot be cancelled even if any other terms are added to (7). This is because the contribution of the bracket cannot be represented by any products of forms due to the extra $\hat{\epsilon}$ but that all the terms of $S_{M 9}^{W Z}$ should be represented by some products of forms.

Our idea to resolve this problem is as follows: Since the main obstruction is the existence of the extra $\hat{\epsilon}$ in (6), let us suppose one rewrite the first bracket of (6) by using the "dual fields" of $\hat{k}_{\hat{\mu}}$ and $\hat{C}$ through duality relations. Then, the extra $\hat{\epsilon}$ is cancelled and the first bracket can be expressed as a sum of exterior products of forms. Thus, it is expected that one can construct a gauge invariant WZ action. This idea is successful, which we show in the following.

The dual field of the 3 -form $\hat{C}$ is the 6 -form $\hat{C}^{(6)}$ whose massive gauge transformation, field strength and the duality relation are 9

$$
\begin{aligned}
\delta \hat{C}_{\mu_{1} \cdots \mu_{6}}^{(6)}= & 30 \partial_{\left[\mu_{1}\right.} \hat{\chi}_{\mu_{2} \mu_{3}} \hat{C}_{\left.\mu_{4} \mu_{5} \mu_{6}\right]} \\
+ & 6 \hat{M} \hat{\lambda}_{\left[\mu_{1}\right.}\left(i_{\hat{k}} \hat{C}^{(6)}\right)_{\left.\mu_{2} \cdots \mu_{6}\right]} \\
\hat{G}_{\mu_{1} \cdots \mu_{7}}^{(7)}= & 7\left[\partial \hat{C}^{(6)}-3 \hat{M}\left(i_{\hat{k}} \hat{C}\right)\left(i_{\hat{k}} \hat{C}^{(6)}\right)\right. \\
+ & 10 \hat{C} \partial \hat{C}+5 \hat{M} C\left(i_{\hat{k}} \hat{C}\right)^{2} \\
& \left.+\frac{\hat{M}}{7}\left(i_{\hat{k}} \hat{N}^{(8)}\right)\right]_{\mu_{1} \cdots \mu_{7}} \\
\hat{G}^{(4) \mu_{1} \cdots \mu_{4}}= & \frac{\epsilon^{\mu_{1} \cdots \mu_{11}}}{7 ! \sqrt{|\hat{g}|}} \hat{G}_{\mu_{5} \cdots \mu_{11}}^{(7)} .
\end{aligned}
$$

$\hat{N}^{(8)}$ is the dual field of the Killing vector also introduced in ref. [9], whose gauge transformation is suggested such as

$$
\begin{aligned}
\delta \hat{N}_{\mu_{1} \cdots \mu_{8}}^{(8)}= & \left\{\frac{8 !}{3 \cdot 4 !} \partial \hat{\chi} \hat{C}\left(i_{\hat{k}} \hat{C}\right)\right. \\
& \left.+8 \hat{M} \hat{\lambda}\left(i_{\hat{k}} \hat{N}^{(8)}\right)\right\}_{\mu_{1} \cdots \mu_{8}}
\end{aligned}
$$

In this paper we regard $\hat{k}_{\mu} \equiv\left(i_{\hat{k}} \hat{g}\right)_{\mu}$ as a "vector gauge field", and consider the "field strength" of

${ }^{2}$ We concentrate our discussions on the gauge transformations with respect to $\hat{\chi}$ and $\hat{\lambda}$. 
it, as one does for $\left(i_{\hat{k}} \hat{C}\right)$. Then, if we define $\hat{G}^{(2)}$ as

$\hat{G}_{\mu \nu}^{(2)} \equiv 2 \partial_{[\mu} \hat{k}_{\nu]}-\hat{M}|\hat{k}|^{2}\left(i_{\hat{k}} \hat{C}\right)_{\mu \nu}$,

$\hat{G}^{(2)}$ is shown to transform covariantly under (1). So, $\hat{G}^{(2)}$, in fact arising in the first term of (6), can be interpreted as the field strength of $\hat{k}_{\mu}$. On the other hand, the field strength $\hat{G}^{(9)}$ of the full 8 -form $\hat{N}^{(8)}$ is difficult to construct. However, in order to rewrite the first term through the duality relation between $\hat{G}^{(9)}$ and $\hat{G}^{(2)}$, it is sufficient to know the field strength of $\left(i_{\hat{k}} \hat{N}^{(8)}\right)$. This is because $\hat{G}^{(2)}$ in the first term of (6) vanishes if any of the indices of $\hat{G}^{(2)}$ takes $z$, implying that one of the indices of $\hat{G}^{(9)}$ certainly takes $z$. Thus, only the field strength of $\left(i_{\hat{k}} \hat{N}^{(8)}\right)$ is needed, and it can be defined as

$$
\begin{aligned}
& \left(i_{\hat{k}} \hat{G}^{(9)}\right)_{\mu_{1} \cdots \mu_{8}} \equiv 8\left\{\partial\left(i_{\hat{k}} \hat{N}^{(8)}\right)\right. \\
& +21\left(i_{\hat{k}} \hat{C}^{(6)}\right) \partial\left(i_{\hat{k}} \hat{C}\right)+35 C \partial\left(i_{\hat{k}} \hat{C}\right)\left(i_{\hat{k}} \hat{C}\right) \\
& \left.+35 \partial C\left(i_{\hat{k}} \hat{C}\right)^{2}+\frac{105}{8} \hat{M}\left(i_{\hat{k}} \hat{C}\right)^{4}\right\}_{\left[\mu_{1} \cdots \mu_{8}\right]} .
\end{aligned}
$$

We note that $\left(i_{\hat{k}} \hat{G}^{(9)}\right)$ is invariant under (1)), which means that this definition is consistent. Then, we assume the duality relation:

$\hat{G}^{(2) \mu_{1} \mu_{2}}=\frac{\epsilon^{\mu_{1} \cdots \mu_{10} z}}{9 ! \sqrt{|\hat{g}|}}\left(i_{\hat{k}} \hat{G}^{(9)}\right)_{\mu_{3} \cdots \mu_{10}}$.

It gives one of the 10D IIA duality relations in ref. 13 on dimensional reduction in $z$, which means that (14) is consistent.

Since all the preparations have been done, let us substitute the relation (10) and (14) for (6) to have the rewritten expression of the massive gauge transformation of $\hat{A}^{(10)}$ :

$$
\begin{aligned}
\delta\left(i_{\hat{k}} \hat{A}^{(10)}\right)_{\mu_{1} \cdots \mu_{9}} & =-9 !\left[\frac{1}{7 !} \partial\left(i_{\hat{k}} \hat{N}^{(8)}\right) \hat{\lambda}\right. \\
& -\frac{1}{2 \cdot 5 !} \partial\left\{\left(i_{\hat{k}} \hat{C}^{(6)}\right)\left(i_{\hat{k}} \hat{C}\right)\right\} \hat{\lambda} \\
& +\frac{1}{6 \cdot 4 !} \partial\left\{\hat{C}\left(i_{\hat{k}} \hat{C}\right)^{2}\right\} \hat{\lambda} \\
& \left.-\frac{\hat{M}}{2^{4} \cdot 4 !}\left(i_{\hat{k}} \hat{C}\right)^{4} \hat{\lambda}\right]_{\left[\mu_{1} \cdots \mu_{9}\right]} \cdot(15)
\end{aligned}
$$

By using this expression, the gauge invariant WZ action of the M-9-brane can be constructed in- deed. Before constructing it, we give the rewritten field equation of $\hat{M}(x)$ :

$$
\begin{gathered}
-\hat{M}|\hat{k}|^{4}=\frac{10 \hat{\epsilon}^{\mu_{1} . . \mu_{10} z}}{10 ! \sqrt{|\hat{g}|}}\left\{\partial_{\mu_{1}}\left(i_{\hat{k}} \hat{A}^{(10)}\right)_{\mu_{2} . . \mu_{10}}\right. \\
-\frac{9 !}{8 \cdot 6 !}\left(i_{\hat{k}} \hat{G}^{(7)}\right)\left(i_{\hat{k}} \hat{C}\right)^{2}+\frac{9 !}{2 \cdot 8 !}\left(i_{\hat{k}} \hat{G}^{(9)}\right)\left(i_{\hat{k}} \hat{C}\right) \\
\left.\quad+\frac{9 !}{288} \partial \hat{C}\left(i_{\hat{k}} \hat{C}\right)^{3}+\frac{9 \cdot 9 !}{5760} \hat{M}\left(i_{\hat{k}} \hat{C}\right)^{5}\right\}_{\mu_{1} . . \mu_{10}} .
\end{gathered}
$$

Since the r.h.s. of $(16)$ is shown to be gauge invariant, it can be interpreted as the gauge invariant field strength of the 10-form (multiplied by $1 / 10$ !). Thus, we can conclude that the 10 -form $\hat{A}^{(10)}$ is introduced consistently. Moreover, we define a new 10 -form $\hat{C}^{(10)}$ which agrees with $10 \mathrm{D}$ IIA 9-form $C^{(9)}$ on dimensional reduction along $\mathrm{z:}$

$$
\begin{aligned}
&\left(i_{\hat{k}} \hat{C}^{(10)}\right)_{\mu_{1} \cdots \mu_{9}} \equiv\left(i_{\hat{k}} \hat{A}^{(10)}\right)_{\mu_{1} \cdots \mu_{9}} \\
&+\left[\frac{9 !}{2 \cdot 7 !}\left(i_{\hat{k}} \hat{N}^{(8)}\right)\left(i_{\hat{k}} \hat{C}\right)-\frac{9 !}{2^{3} 5 !}\left(i_{\hat{k}} \hat{C}^{(6)}\right)\left(i_{\hat{k}} \hat{C}\right)^{2}\right. \\
&\left.+\frac{9 !}{2^{4}(3 !)^{2}} \hat{C}\left(i_{\hat{k}} \hat{C}\right)^{3}\right]_{\left[\mu_{1} . . \mu_{9}\right]}
\end{aligned}
$$

Then, the gauge transformation of $\hat{C}^{(10)}$ takes the simple form:

$$
\begin{aligned}
\delta\left(i_{\hat{k}} \hat{C}^{(10)}\right)_{\mu_{1} \cdots \mu_{9}}= & -945\left\{-4 \partial \hat{\chi}\left(i_{\hat{k}} \hat{C}\right)^{3}\right. \\
& \left.+\hat{M}\left(i_{\hat{k}} \hat{C}\right)^{4} \hat{\lambda}\right\}_{\left[\mu_{1} \cdots \mu_{9}\right]} .
\end{aligned}
$$

For convenience, we use $\hat{C}^{(10)}$ to construct $S_{\mathrm{M} 9}^{W Z}$.

Now, we construct the M-9-brane WZ action as that of the gauged $\sigma$-model, in which the translation along $\hat{k}$ is gauged 14 [15]. In this approach the M-9-brane wrapped around the compact isometry direction is described 10. So, denoting its worldvolume coordinates by $\xi^{i}(i=$ $0,1, . ., 8)$ and their embeddings by $X^{\mu}(\xi)(\mu=$ $0,1, . ., 9, z)$, the worldvolume gauge transformation is given by $\delta_{\eta} X^{\mu}=\eta(\xi) \hat{k}^{\mu}$ where $\eta(\xi)$ is a scalar gauge parameter. In order to make the brane action invariant under the transformation, the derivative of $X^{\mu}$ with respect to $\xi^{i}$ is replaced by the covariant derivative $D_{i} X^{\mu}=\partial_{i} X^{\mu}-\hat{A}_{i} \hat{k}^{\mu}$ with the gauge field $\hat{A}_{i}=-|\hat{k}|^{-2} \partial_{i} \hat{X}^{\hat{\nu}} \hat{k}_{\hat{\nu}}[15]$. Then, we obtain the M-9-brane WZ action only on the basis of the gauge invariance, as

$$
S_{M 9}^{W Z}=T_{\mathrm{M} 9} \int d^{9} \xi \epsilon^{i_{1} \cdots i_{9}}\left[\frac{1}{9 !}\left(i_{\hat{k}} \widetilde{\hat{C}^{(10)}}\right)_{i_{1} \cdots i_{9}}\right.
$$




$$
\begin{array}{r}
+\frac{1}{2 \cdot 7 !}\left(\widetilde{i}_{\hat{k}}^{\hat{N}^{(8)}}\right)_{i_{1} \cdots i_{7}} \hat{\mathcal{K}}_{i_{8} i_{9}}^{(2)} \\
+\frac{1}{2^{3} \cdot 5 !}\left(\widetilde{(i \hat{k}}_{\hat{\hat{C}}(6)}^{(6)}\right)_{i_{1} \cdots i_{5}}\left(\hat{\mathcal{K}}^{(2)}\right)_{i_{6} \cdots i_{9}}^{2} \\
+\frac{1}{2 \cdot(3 !)^{2}} \widetilde{\hat{C}}_{i_{1} i_{2} i_{3}}\left\{(\partial \hat{b})^{2}-\frac{1}{4}\left(\widetilde{i_{\hat{k}} \hat{C}}\right) \partial \hat{b}\right. \\
\left.+\frac{1}{8}\left(\widetilde{i_{\hat{k}} \hat{C}}\right)^{2}\right\}_{i_{4} \cdots i_{7}} \hat{\mathcal{K}}_{i_{8} i_{9}}^{(2)} \\
+\frac{1}{2 \cdot 4 !} \hat{A}_{i_{1}}\left\{(\partial \hat{b})^{3}+\frac{1}{2}(\partial \hat{b})^{2}\left(\widetilde{i_{\hat{k}} \hat{C}}\right)\right. \\
\left.+\frac{1}{4}(\partial \hat{b})\left(\widetilde{i_{\hat{k}} \hat{C}}\right)^{2}+\frac{1}{8}\left(\widetilde{i_{\hat{k}} \hat{C}}\right)^{3}\right\}_{i_{2} \cdots i_{7}}\left(\hat{\mathcal{K}}^{(2)}\right)_{i_{8} i_{9}} \\
\left.+\frac{m}{5 !} \hat{b}_{i_{1}}(\partial \hat{b})_{i_{2} \cdots i_{9}}^{4}\right]
\end{array}
$$

where $\widetilde{\hat{S}}_{i_{1} \cdots i_{r}} \equiv \hat{S}_{\mu_{1} \cdots \mu_{r}} D_{i_{1}} X^{\mu_{1}} \cdots D_{i_{r}} X^{\mu_{r}}$ for a target-space field $\hat{S}_{\mu_{1} \cdots \mu_{r}}$. $\hat{b}_{i}$ describes the flux of an M-2-brane wrapped around the isometry direction, whose massive gauge transformation is determined by the requirement of the invariance of its modified field strength $\hat{\mathcal{K}}_{i j}^{(2)}=2 \partial_{[i} \hat{b}_{j]}-$ $\partial_{i} X^{\mu} \partial_{j} X^{\nu}\left(i_{\hat{k}} \hat{C}\right)_{\mu \nu}$ (i.e. $\delta \hat{b}_{i}=\hat{\lambda}_{i}$ ).

Then, we check the consistency of the M-9brane action in two ways; (the kinetic term of the M-9-brane has been given in ref.[16].) first, we can improve the M-9-brane solution in ref. 110 so that $\hat{A}^{(10)} \neq 0$. Then, the M-9-brane worldvolume action must be the source of the solution. We can show that this is true 11. Second, when there are two M-9-branes parallel to each other with a certain orientation, no force exists between them, so, the potential energy of a test M-9-brane parallel to a background M-9-brane must vanish. Using the obtained M-9-brane action and the improved M-9-brane solution, we can show that this is also true 11]. Thus, we can say that the obtained M-9-brane action is consistent.

Finally, we present the result of dimensional reductions of $S_{M 9}^{W Z}$ briefly. First, if we consider the dimensional reduction along the isometry direction, it is shown to give the D-8-brane WZ action. Second, if we consider the dimensional reduction along the only transverse direction, $S_{M 9}^{W Z}$ is shown to give the NS-9A-brane WZ action. (In fact, in this case, we need to know "undiscussed" truncation conditions caused by modding out the system by an certain $Z_{2}$ symmetry, but we can infer them by using the duality relations 10 and (14).) Thus, the relation of p-branes with $p \geq 8$, based on the superalgebra, is consistent from the viewpoint of their WVEAs.

\section{Summary and discussion}

The results of this work is summarized as follows: The M-9-brane Wess-Zumino action, the only unconstructed (bosonic part of) brane action, has been obtained, based only on the gauge invariance. The essential point in constructing it is our appropriate choice of fields representing the same degrees of freedom. Its consistency has been confirmed in two ways. In addition, upon two kinds of dimensional reductions, the WessZumino action of the M-9-brane has been shown to give those of the D-8-brane and the NS-9A brane, respectively. Therefore, we conclude that within the framework of massive 11D theory, the relation of p-branes with $p \geq 8$, suggested on the basis of superalgebra, is consistent from the viewpoint of their worldvolume effective actions.

In this theory, however, the implication of the existence of the isometry direction is still unclear, so some other modification of the framework might be needed.

Finally, we would like to note that there is third possibility of dimensional reduction of the M-9brane; the dimensional reduction along the worldvolume direction but not the isometry one. There are some arguments on how to interpret this possibility, and ours is that the obtained 8-brane is essentially the same as the usual D-8-brane except that it arises in another massive extension of the 10-dimensional IIA theory with an isometry direction. Since we do not have enough space to discuss it here, in detail, please see ref.12 and references therein.

\section{Acknowledgment}

I would like to thank Taro Tani, Tunehide Kuroki and Shinya Tamura for fruitful discussions and encouragement in completing the work in refs. 11] 12]. I am grateful to Professor Eric Bergshoeff for useful comments and Yolanda Lozano for useful comments on the work 12] via email. I am especially grateful to Professor Dmitri 
Sorokin, Professor Alexei Nurmagambetov and all the other staffs and students supporting the conference, for inviting me to the conference and taking much care of me and my wife very kindly before and during the conference. I am also grateful to Yoshida Foundation for Science and Technology for partial financial support.

\section{REFERENCES}

1. C. M. Hull, Nucl. Phys. B509 (1998) 216, hep-th/9705162.

2. P. K. Townsend, "M-theory from its Superalgebra", Cargese Lectures 1997, hepth/9712004.

3. P. K. Townsend, "P-brane Democracy", hepth/9507048.

4. E. Bergshoeff, E. Eyras, R. Halbersma, C. M. Hull, Y. Lozano and J. P. van der Schaar, Nucl. Phys. B564 (2000) 29, hep-th/9812224.

5. J. Polchinski, Phys. Rev. Lett. 75 (1995) 4724, hep-th/9510017.

6. J. Polchinski and E. Witten, Nucl. Phys. B460 (1996) 525, hep-th/9510169.

7. E. Bergshoeff, M. de Roo, M. B. Green, G. Papadopoulos and P. K. Townsend, Nucl. Phys. B470 (1996) 113, hep-th/9601150.

8. K. Bautier, S. Deser, M. Henneaux and D. Seminara, Phys. Lett B406 (1997) 49, hepth/9704131.

9. E. Bergshoeff, Y. Lozano and T. Ortin, Nucl. Phys. B518 (1998) 363, hep-th/9712115.

10. E. Bergshoeff and J. P. van der Schaar, Class. Quant. Grav. 16 (1999) 23, hep-th/9806069.

11. T. Sato, Phys. Lett. B 477 (2000) 457, hepth/9912030.

12. T. Sato, "On dimensional reductions of $M-9$ branes", hep-th/0003240.

13. M. B. Green, C. M. Hull and P. K. Townsend, Phys. Lett. B382 (1996) 65, hep-th/9604119.

14. E. Bergshoeff, B. Janssen and T. Ortin, Phys. Lett. B410 (1997) 131, hep-th/9706117.

15. E. Bergshoeff, E. Eyras and Y. Lozano, Phys. Lett. B430 (1998) 77, hep-th/9802199.

16. E. Eyras and Y. Lozano, "Brane Actions and String Dualities", hep-th/9812225. 